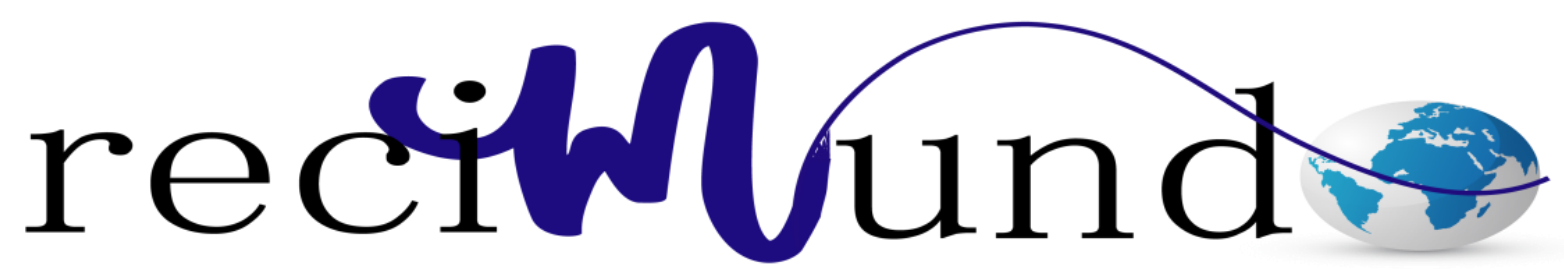

Revista Cientifica Mundo de la Investigación y el Conocimiento

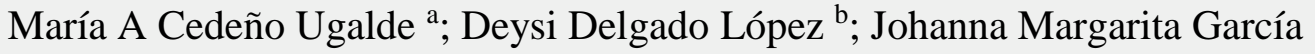 \\ Quiroz $^{\text {c; }}$ Sandra Jazmín Muñiz Tóala ${ }^{\text {d; Liliana Maribel Pionce Sánchez }}{ }^{\text {e; Tatiana }}$ \\ Lissette Marcillo Quimis ${ }^{\mathrm{f}}$
}

La administración de la salud y la calidad del desempeño para los beneficiarios

Health management and quality performance for beneficiaries

Revista Científica Mundo de la Investigación y el Conocimiento. Vol. 2 núm.4,

Octubre, ISSN: 2588-073X, 2018, pp. 160-188

DOI: 10.26820/recimundo/2.(4).octubre.2018.160-188

URL: http://www.recimundo.com/index.php/es/article/view/349

Editorial Saberes del Conocimiento

Recibido: 15/07/2018

Aceptado: 05/08/2018

Publicado: 30/10/2018

a. Doctora en Medicina y Cirugía, Magister en Epidemiologia, Docente Universidad Laica Eloy Alfaro de Manabí - Facultad de Ciencias Médicas - Carrera de Medicina.

b. Licenciada en Enfermería, Magister en Gerencia en Salud para el Desarrollo Local; Docente Universidad Estatal del Sur de Manabí, Facultad Ciencias de la Salud, Carrera de Enfermería.

c. Licenciada en Enfermería, Magister en Emergencias Médicas, Docente de la Universidad Técnica de Manabí, Hospital Verdi Cevallos Balda.

d. Licenciada en Enfermería, Hospital IESS - Jipijapa; Responsable de Consulta externa.

e. Licenciada en Enfermería, Hospital de Especialidades de Portoviejo - Pediatría.

f. Licenciada en Enfermería, Hospital General Manta IESS. 


\section{La administración de la salud y la calidad del desempeño para los \\ beneficiarios}

Vol. 2, núm. 4., (2018)

María A Cedeño Ugalde; Deysi Delgado López; Johanna Margarita García Quiroz; Sandra Jazmín Muñiz Tóala; Liliana Maribel Pionce Sánchez; Tatiana Lissette Marcillo Quimis

\section{RESUMEN}

En el presente trabajo se explora la administración de la salud y la calidad del desempeño para los beneficiarios. La administración posee los elementos necesarios que aplicados al área de la salud, estos van a contribuir en el desarrollo organizacional de la institución de salud a fin de desarrollar una visión en conjunto del sistema optimizando los recursos económicos, humanos y técnicos con la intención de mejora el desempeño de la institución de salud hacia un crecimiento de calidad eficaz y eficiente. En que la atención de la salud debe ser de la forma más humana posible de tal forma que también asegure la participación activa de los beneficiarios como parte del desempeño mientras reciben los servicios de salud consecuentemente respetando la lealtad y libertad del paciente, a fin de cumplir con el consentimiento informando todo lo necesario y estableciendo una adecuada relación médico paciente, por tanto es imprescindible el adecuado control en busca de la mejor calidad, teniendo en cuenta que los profesionales y trabajadores de la salud conocen bien los problemas y cuáles son sus causas y, por consiguiente cual es la solución, por tanto la atención de estas particularidades de la salud deben ser comprendidas por los directivos y trabajadores de la salud, además es necesario tener presente que hay que conocer las expectativas de los beneficiarios a quienes se les brinda los servicios de salud de calidad. Consiguientemente las instituciones de salud deben cumplir su misión orientada hacia la satisfacción de los pacientes beneficiarios, cumpliendo con los reglamentos, procedimientos y normas institucionales.

Palabras claves: Administración de la salud, desarrollo organizacional, lealtad y libertad del paciente, salud de calidad, normas institucionales. 


\section{La administración de la salud y la calidad del desempeño para los beneficiarios}

Vol. 2, núm. 4., (2018)

María A Cedeño Ugalde; Deysi Delgado López; Johanna Margarita García Quiroz; Sandra Jazmín Muñiz Tóala; Liliana Maribel Pionce Sánchez; Tatiana Lissette Marcillo Quimis

\section{ABSTRACT}

In the present work, the administration of health and the quality of performance for the beneficiaries is explored. The administration has the necessary elements that applied to the area of health, these will contribute to the organizational development of the health institution in order to develop a vision of the system as a whole, optimizing the economic, human and technical resources with the intention of improving the performance of the health institution towards an effective and efficient quality growth. That health care should be as human as possible in such a way that it also ensures the active participation of the beneficiaries as part of the performance while receiving health services consistently respecting the patient's loyalty and freedom, in order to fulfill with the consent informing all the necessary and establishing an adequate patient medical relationship, therefore it is essential the adequate control in search of the best quality, taking into account that professionals and health workers know the problems well and what are their causes and, therefore what is the solution, therefore the attention of these particularities of the health must be understood by the managers and workers of the health, in addition it is necessary to bear present that it is necessary to know the expectations of the beneficiaries to whom the quality health services. Consequently, the health institutions must fulfill their mission oriented towards the satisfaction of the beneficiary patients, complying with the regulations, procedures and institutional norms.

Keywords: Health administration, organizational development, patient loyalty and freedom, quality health, institutional norms. 


\section{La administración de la salud y la calidad del desempeño para los \\ beneficiarios}

Vol. 2, núm. 4., (2018)

María A Cedeño Ugalde; Deysi Delgado López; Johanna Margarita García Quiroz; Sandra Jazmín Muñiz Tóala; Liliana Maribel Pionce Sánchez; Tatiana Lissette Marcillo Quimis

\section{Introducción.}

El administrar los de servicios de salud de manera ideal, es lo más evidente que le corresponde y merece ser operado por el sector estatal, lamentablemente los resultados no han sido los proyectados. Cuando a nivel mundial se vive una crisis en el sector y los servicios de calidad los ha entregado el sector privado pero con un delicado problema que es el fraccionamiento de las personas las mismas que logran alcanzar estos servicios médicos hospitalarios, los que están inmersos en una controversia ética, que imposibilita satisfacer en igualdad en lo que respecta a la oferta y la demanda con relación a los costos. Germinando los problemas con más fuerza, con malos servicios públicos de deficiente calidad para los beneficiarios.

El hecho que el hombre exista como parte de un colectivo lleva al ser humano a la necesidad vital de relacionarse en la creación de pequeñas y grandes obras, como las pirámides de Egipto, que son inconcebibles sin una conducción, lo cual quiere decir que siempre existió de alguna forma la administración.

La administración ha sido el resultado de un proceso iniciado en los albores de la humanidad y evolucionado en diferentes épocas y etapas: en China, Egipto, Roma, la época feudal, en Francia con Montesquieu, sin olvidar las religiones. Es interesante la referencia del episodio relatado en el libro Éxodo de la Biblia, hace aproximadamente 3000 años, cuando el suegro de Moisés, Jetro, lo visita en el desierto y le aconseja que en la comunicación con el pueblo lo primero es educarles y enseñarles el camino, y que para juzgar los asuntos y no desfallecer él y todos los demás por el tiempo invertido en esos menesteres, debía escoger a 


\section{La administración de la salud y la calidad del desempeño para los beneficiarios}

Vol. 2, núm. 4., (2018)

María A Cedeño Ugalde; Deysi Delgado López; Johanna Margarita García Quiroz; Sandra Jazmín Muñiz Tóala; Liliana Maribel Pionce Sánchez; Tatiana Lissette Marcillo Quimis

hombres de virtud como jefes y jueces "sobre mil, sobre ciento, sobre cincuenta, y sobre diez", quienes juzgarían los asuntos pequeños, llevando a él solo los importantes. Esto se considera el antecedente más remoto de la técnica de delegación de autoridad en la administración, (levítico), Díaz, (1988).

Históricamente en el seno de las sociedades los hombres sintieron la necesidad vital de relacionarse, pero esas relaciones eran inconcebibles sin alguien que las dirigiera, teniendo en cuenta la interdependencia de las múltiples organizaciones religiosas, y las industrias. Las teorías y escuelas de administración como ciencia realmente surgen como una necesidad después del surgimiento del capitalismo en el siglo XVII y el advenimiento de la Revolución Industrial a fines del siglo XVIII. La administración como ciencia surgió a finales del siglo XIX con Frederick Taylor y otros autores, aunque se reconoce que el verdadero científico y creador lo fue el francés Henry Fayol que en 1912 la definió como ciencia universal, estableció sus reglas y principios, y la estructuro en cinco funciones, que en estos momentos se las denomina procesos administrativos, las cuales nombró como: planeamiento, organización, coordinación, autoridad y control, Ramos, (2011).

Los principios generales y las funciones clásicas de la administración de Fayol son aún concepciones vigentes. Se le considera el verdadero científico o padre de la Administración como ciencia. Entre sus trabajos esta su publicación en Francia, 1916, de Administración industrial y general que se tradujo mucho después y fue publicado en los EE.UU. en 1929. La escuela de administración científica, cuyo fundador fue el norteamericano Frederick W. Taylor 


\section{La administración de la salud y la calidad del desempeño para los \\ beneficiarios}

Vol. 2, núm. 4., (2018)

María A Cedeño Ugalde; Deysi Delgado López; Johanna Margarita García Quiroz; Sandra Jazmín Muñiz Tóala; Liliana Maribel Pionce Sánchez; Tatiana Lissette Marcillo Quimis

(1856-1915) y se incluyen otros científicos como Henry L. Gantt (1861-1919), Frank B. (1868-

1924) y Lillian M. Gilbert (1878-1972), Ramos, (2011).

Taylor dirigió sus estudios al trabajo por cronometraje en la línea de producción. Estableció normas para la cantidad de trabajo que se ha de realizar y sugirió que se pagara extra a los obreros más productivos con un sistema de tasas diferenciadas. Esta escuela surgió por la necesidad de aumentar la productividad de los trabajadores y su método exigía que estos cumplieran las normas técnicas y laborales de los especialistas. La calidad dependía de la inspección y consideraba que los trabajadores se limitaban a seguir las órdenes. Ramos, (2011).

Tener una Administración Pública alineada a la calidad, tiene como meta principal que todas sus organizaciones trabajen de ese modo, al coordinar que cada una de ellas pueda optar por aplicar un modelo de gestión, que le permita mediante el propio esfuerzo, mejorar de un modo continuo la atención de los ciudadanos. Casermeiro et al, (s/f).

El Estado invierte mucho dinero en el bienestar de sus ciudadanos, haciendo frente a las difíciles condiciones económicas y sociales que suponen los tiempos que corren. Se trata de todo un desafío, porque el saber afrontar el profundo impacto que producen las nuevas modalidades y las nuevas tecnologías que se van incorporando, posibilitan brindar una mejor atención y una mayor calidad en las prestaciones públicas. Por esta sencilla razón, la calidad en los servicios públicos tiene un papel fundamental. Sobre todo en lo que al ahorro se refiere: porque lleva implícita un ahorro de tiempo, de trabajo, de elementos de trabajo, que se traduce en menores costos y redunda en beneficio colectivo. Casermeiro et al, (s/f). 


\section{La administración de la salud y la calidad del desempeño para los beneficiarios}

Vol. 2, núm. 4., (2018)

María A Cedeño Ugalde; Deysi Delgado López; Johanna Margarita García Quiroz; Sandra Jazmín Muñiz Tóala; Liliana Maribel Pionce Sánchez; Tatiana Lissette Marcillo Quimis

La clave de una gestión acertada en el desarrollo de las organizaciones está en las personas que participan en ella. En el mundo actual los altos niveles de competitividad exigen nuevas formas de compromiso, de ver los hechos, de decidir y dirigir, de pensar y sentir, así como de desarrollar los diferentes procesos en las organizaciones de una forma más efectiva, Mora, (2005).

En la actualidad, la administración en los sistemas de salud requiere un conjunto de conocimientos, habilidades y técnicas con base en un equilibrio armónico que proporcione la preparación suficiente al directivo y le permita llevar a cabo una secuencia de acciones para alcanzar objetivos determinados en la solución de problemas prioritarios. Un administrador en salud debe tener una visión holística e integral del sistema; por ello, su visión y quehacer rebasa la administración de una clínica, hospital, unidad médica o centro de salud; debe ser capaz del diseño (planeación), desarrollo (operación), sistematización (orden y registro), evaluación y retroalimentación (análisis y reorientación de acciones y metas) de los planes y programas de trabajo de los mismos, Morales, (2013).

Es indudable el interés que existe sobre la administración al proceso de control de calidad, por supuesto este se basa en garantizar la calidad que es una obligación para todos los que administran y tienen que prestar servicios en este caso de salud, puesto que es una necesidad imprescindible en el perfeccionamiento para quienes están involucrados en la prestación de dichos servicios públicos, en que precisamente el control de calidad es el objetivo principal en la administración.

\section{Metodología.}




\section{La administración de la salud y la calidad del desempeño para los \\ beneficiarios}

Vol. 2, núm. 4., (2018)

María A Cedeño Ugalde; Deysi Delgado López; Johanna Margarita García Quiroz; Sandra Jazmín Muñiz Tóala; Liliana Maribel Pionce Sánchez; Tatiana Lissette Marcillo Quimis

En la presente investigación científica se realizó un análisis de la bibliografía de la literatura más cercana sobre la administración de la salud y la calidad del desempeño para los beneficiarios. Para la realización de la actual investigación, se consideró, información confiable sobre trabajos plasmados, tratados en este tema, se utilizó información bibliográfica, consultas electrónicas, misma que se la obtuvo de libros electrónicos, artículos científicos y revistas.

\section{Desarrollo.}

La administración o gerencia de los servicios de sanitarios

La administración en salud o administración sanitaria es la ciencia social y técnica relacionada con la planificación, organización, dirección y control de las empresas públicas y privadas del sector salud, mediante la optimización de recursos financieros, tecnológicos y humanos. En otras palabras, la administración en salud es la administración de empresas aplicada a las empresas proveedoras de bienes y servicios de salud, que resulten en la recuperación o mejora en la salud de las personas usuarias, a quienes -dentro del establecimiento de salud- se les denominan clientes y/o pacientes. A la administración en salud también se la conoce con los nombres de gerencia en salud, gestión sanitaria, gestión clínica, administración de empresas de salud, administración de servicios de salud y gerencia de servicios de salud, Morales, (2013).

La administración en sentido formal, es aquella que se realiza en una organización, la cual posee cuatros funciones específicas que son: la planificación, la organización, la dirección y el control; estas en conjunto se conocen como proceso administrativo y se puede definir como las 


\section{La administración de la salud y la calidad del desempeño para los beneficiarios}

Vol. 2, núm. 4., (2018)

María A Cedeño Ugalde; Deysi Delgado López; Johanna Margarita García Quiroz; Sandra Jazmín Muñiz Tóala; Liliana Maribel Pionce Sánchez; Tatiana Lissette Marcillo Quimis

diversas funciones que se deben realizar para que se logren los objetivos con la óptima utilización de los recursos en una institución, Romero, (2010).

La administración funcional se aplica a la organización vertical tradicional mediante la planificación estratégica, las directrices, el establecimiento de las rutinas o actividades fundamentales que se han de realizar en el trabajo diario y la elaboración de los procedimientos para cumplimentarlas, cuya finalidad es estabilizar el sistema y, posteriormente, perfeccionarlo. Sin embargo, desde hace mucho tiempo se ha venido planteando que con la administración funcional se presentan, en ocasiones, dificultades en la solución de problemas y el cumplimiento de las tareas complejas debido a la falta de coordinación que se observa entre las divisiones, los niveles administrativos medios, departamentos y secciones, Ramos, (2011).

Un proceso o ciclo es el conjunto de pasos o etapas necesarios para llevar a cabo una actividad o lograr un objetivo. Ciclo administrativo tiene dos fases (mecánica y dinámica) y cuatro etapas (planificación, organización, dirección y control) sucesivas a través de las cuales se efectúa la administración, mismas que se interrelacionan y forman un proceso integral en el cual cada etapa incluye a las otras para poder ejecutarse, MSPAS, (1997).

Las organizaciones tienen una finalidad, objetivos de supervivencia; pasan por ciclos de vida y enfrentan problemas. Tienen una personalidad, una necesidad, un carácter y se consideran como microsociedad, con proceso de socialización, normas y su propia historia. Todo esto relacionado con el clima, la imagen, la cultura y la gestión organizacional que en los últimos años han tenido el reconocimiento de la importante función que desempeñan en el desarrollo de la organización, Minsal y Pérez, (2007). 


\section{La administración de la salud y la calidad del desempeño para los \\ beneficiarios}

Vol. 2, núm. 4., (2018)

María A Cedeño Ugalde; Deysi Delgado López; Johanna Margarita García Quiroz; Sandra Jazmín Muñiz Tóala; Liliana Maribel Pionce Sánchez; Tatiana Lissette Marcillo Quimis

El desarrollo de las actividades en los servicios de Salud es un proceso de alta complejidad, ya que no se relaciona únicamente con alcanzar los resultados esperados en la planificación, sino que tiene que ver con todos los elementos, visibles y no visibles, que deben darse para lograr dichos resultados. Superados los primeros momentos de la gestión en los servicios (definir objetivos generales, proyectos, asignar recursos y personal) es indispensable garantizar el cumplimiento de lo resuelto, momento en el que surge una serie de complicaciones y obstáculos de carácter logístico y de naturaleza humana que a veces llegan hasta a neutralizar la acción en las unidades operativas, Morales, (2013).

El control es un elemento del proceso administrativo que incluye todas las actividades que se emprenden para garantizar que las acciones reales coincidan con las acciones planificadas. Todos los directivos de una organización tienen la obligación de controlar, de tal manera, el control es un elemento clave en la gestión organizacional. Romero, (2010).

La Administración Pública es imprescindible y que debemos aspirar a la mejor posible. Se puede cuestionar su tamaño, su papel o su funcionamiento, pero no cabe duda de que no podemos vivir sin el sector público. Se puede y se debe discutir si lo público responde a las necesidades de los ciudadanos o si está adaptado a un mundo en pleno cambio, pero esto no significa que la Administración deje de tener un papel clave en las sociedades del siglo XXI, PwC, (2012).

La administración de servicios de salud es una de las disciplinas de la salud pública. Su función es la integración eficiente y efectiva de los recursos humanos, físicos y económicos para lograr una atención óptima de servicios de salud al paciente. El/la administrador/a procura que 


\section{La administración de la salud y la calidad del desempeño para los beneficiarios}

Vol. 2, núm. 4., (2018)

María A Cedeño Ugalde; Deysi Delgado López; Johanna Margarita García Quiroz; Sandra Jazmín Muñiz Tóala; Liliana Maribel Pionce Sánchez; Tatiana Lissette Marcillo Quimis

los recursos disponibles para la promoción, protección y restauración de la salud sean aplicados tomando en consideración el conocimiento científico vigente sobre la salud y la enfermedad. Morales, (2013).

Hoy la Administración Pública presta servicios públicos concretos en el marco de amplias políticas generales, persiguiendo que las prestaciones se adapten a cada necesidad en particular y que, en determinados casos, potencien la igualdad de las oportunidades de las personas, para que todas ellas alcancen con plenitud el goce de los derechos y libertades fundamentales que la Constitución, los tratados internacionales y las leyes les reconocen. Casermeiro et al, (s/f).

Es la capacidad de gestionar, administrar y financiar las empresas e instituciones de salud, adecuarlas a las exigencias dadas por los nuevos sistemas de prestación de servicios, actualizar su infraestructura y dotación donde se requiera, ajustarse a los procesos de descentralización y modernización de los servicios públicos, mejorar la prevención, promoción, diagnóstico y atención en salud, aplicar los mecanismos tendientes a mejorar la calidad, eficiencia y coberturas de los servicios en todos los niveles, Morales, (2013).

Las administraciones, a su vez, se identifican con las fuerzas políticas que gobiernan, de manera que el papel técnico de los cuadros, profesionales y trabajadores está condicionado por las peculiaridades que cada opción política y, a menudo, la posición particular de los responsables políticos imprime a la tarea ejecutiva que le es propia. Desde luego, éstos tienen, en un régimen democrático, la representatividad de la población o, cuanto menos, de los electores, de manera que cabría esperar diferencias en la administración de los servicios sanitarios en 


\section{La administración de la salud y la calidad del desempeño para los \\ beneficiarios}

Vol. 2, núm. 4., (2018)

María A Cedeño Ugalde; Deysi Delgado López; Johanna Margarita García Quiroz; Sandra Jazmín Muñiz Tóala; Liliana Maribel Pionce Sánchez; Tatiana Lissette Marcillo Quimis

general, y de la SP en particular, según la orientación del partido político en el poder, Segura, (2002).

Según Ramos, (2011), ¿Qué debe hacer la dirección o administración en relación con la calidad?, en primer lugar lograr que todos los trabajadores conozcan la política de calidad de la institución, estar constantemente en contacto con todos los trabajadores y otros directivos. Debe realizar el control administrativo diario, importante para mejorar la calidad, allí mismo donde se realizan las acciones y servicios; además el control de calidad, cuyo objetivo es verificar cómo se está cumpliendo el plan acordado, y medir si el desempeño se ajusta a lo acordado; y conocer las opiniones de todos, trabajadores, responsables y de los que reciben los servicios y, en caso necesario, tomar las medidas correctivas.

La puesta en marcha de nuevos modelos y enfoques en dirección, para el logro de una gestión exitosa en las instituciones de salud, implica una gran responsabilidad social, es decir todos los actores deben estar involucrados en la actividad, unido a un liderazgo efectivo, aprendizaje y desarrollo del talento humano, donde el trabajo en equipo, la planificación y el control se conviertan en garantía para asegurar que los objetivos y planes de la organización se lleven a cabo de forma participativa, Segredo, et al., (2012).

La administración posee herramientas que, aplicadas al ramo de los sistemas de salud, pueden ayudar a éstos a desarrollar una visión de conjunto del sistema, optimizando recursos humanos, técnicos y financieros, para poder llevar a las instituciones de salud a un estado de crecimiento y eficiencia. Frecuentemente, los médicos destacados en su especialidad son llamados a administrar en los distintos niveles jerárquicos del sistema de salud, careciendo las 


\section{La administración de la salud y la calidad del desempeño para los beneficiarios}

Vol. 2, núm. 4., (2018)

María A Cedeño Ugalde; Deysi Delgado López; Johanna Margarita García Quiroz; Sandra Jazmín Muñiz Tóala; Liliana Maribel Pionce Sánchez; Tatiana Lissette Marcillo Quimis

más de las veces de experiencia y preparación específica en administración. también encontramos directivos que son expertos en administración, pero que desconocen los problemas de salud. Finalmente se destaca la necesidad de dar capacitación administrativa al médico, ya que la razón de ser de las instituciones que conforman el sistema de salud es precisamente el servicio a esta última, por lo que las decisiones importantes deben ser tomadas por médicos, Morales, (2013).

MSPAS, (1997). El desarrollo Institucional agrupa las acciones para mejorar la capacidad operativa del sistema de salud en la entrega de servicios, entre las cuales están el establecimiento de sistemas de planificación y programación y presupuesto, la supervisión, seguimiento y evaluación continua, la investigación, la gestión de la calidad. Para la entrega de más y mejores servicios de salud y la gestión por resultados alineando los recursos y las acciones hacia el logro de los efectos deseados. Construyendo así un modelo institucional de gestión por resultados.

Estas mejoras favorecen a que la asignación de recursos se vincule con: $i$ ) la producción generada; ii) a que se le dé seguimiento al cumplimiento de objetivos y metas; iii) a medir el desempeño a través de indicadores y parámetros objetivos; y iv) a que las unidades asuman la responsabilidad de ejercer la conducción y el control para obtener resultados, identificar brechas, investigar sus causas y poner en marcha planes para el mejoramiento continuo, MSPAS, (1997).

Segura, (2002). Una visión de la administración como el brazo ejecutor de las directrices y de las decisiones políticas que es lógico que varíen con la alternancia democrática, parece excesivamente simplista. Ni que fuera sólo porque las reglas del juego democrático no excluyen las aportaciones técnicas o profesionales. Pero es que, en cierto sentido, estas reglas están por 


\section{La administración de la salud y la calidad del desempeño para los \\ beneficiarios}

Vol. 2, núm. 4., (2018)

María A Cedeño Ugalde; Deysi Delgado López; Johanna Margarita García Quiroz; Sandra Jazmín Muñiz Tóala; Liliana Maribel Pionce Sánchez; Tatiana Lissette Marcillo Quimis

encima de los intereses particulares de los políticos y del personal de la administración. Tanto unos como otros deben respetarlas, ya que sin ellas la legitimidad social pierde sus referencias.

Así, los conocimientos y las habilidades del personal de las administraciones podría significar una garantía frente a eventuales arbitrariedades políticas, el valor añadido que justifica su existencia más allá de las organizaciones específicamente políticas cuyo principal objetivo es acceder al poder para gestionarlo precisamente, con la contribución de las administraciones. Para ello es imprescindible una clara delimitación de los ámbitos político y técnico de las actividades de la administración, difícil de conseguir si los procedimientos de acceso a los puestos de trabajo no garantizan la competencia y no se cuenta con una administración pública suficientemente profesionalizada. En caso contrario los profesionales se ven abocados a representar un papel que, según las circunstancias, será el de rehén o el de cómplice, Segura, (2002).

Para Morales, (2013). La Administración de la salud necesita de profesionales que cuenten además de los conocimientos gerenciales modernos, herramientas, involucrados con la salud, el garantizar que las instituciones, organizaciones comprometidas con este servicio ofrezcan calidad gracias a una buena productividad. Se requiere de profesionales comprometidos con la gerencia de la salud que cuenten como señalan algunas universidades, escuelas relacionadas con la Administración de la salud con el poder:

Desarrollar la capacidad de gestión, administrativa y financiera de las empresas e instituciones de salud, adecuarlas a las exigencias dadas por los nuevos sistemas de prestación de servicios, actualizar su infraestructura y dotación donde se requiera, ajustarse a los procesos de descentralización y modernización de los servicios públicos, mejorar la prevención, promoción, 


\section{La administración de la salud y la calidad del desempeño para los beneficiarios}

Vol. 2, núm. 4., (2018)

María A Cedeño Ugalde; Deysi Delgado López; Johanna Margarita García Quiroz; Sandra Jazmín Muñiz Tóala; Liliana Maribel Pionce Sánchez; Tatiana Lissette Marcillo Quimis

diagnóstico y atención en salud, aplicar los mecanismos tendientes a mejorar la calidad, eficiencia y coberturas de los servicios en todos los niveles, Morales, (2013).

La Administración no puede prestar los servicios de la misma manera que antes porque la ciudadanía exige otro tipo de relación. Hoy se pide mayor agilidad en los trámites y más flexibilidad orgánica y funcional en todos los ámbitos en los que lo público interactúa con la ciudadanía. De manera legítima, los ciudadanos esperan que los servicios públicos funcionen correctamente, sin prestar mayor atención a si estos proceden de la Administración local, autonómica o central, porque, para ellos, lo realmente importante es satisfacer sus necesidades y hacer uso de los recursos comunes. PwC, (2012).

Casermeiro et al., (s/f). El crecimiento de los Estados en general ha supuesto que las Administraciones Públicas se hagan cargo de la prestación de una diversidad de servicios, pasando de una primera etapa cuantitativa (para satisfacer las demandas del conjunto de los usuarios), a otra cualitativa (para conseguir más financiación y aumentar su prestigio), la cual redunda en la búsqueda de una mayor calidad para sus servicios.

\section{Reingeniería}

Reingeniería es el diseño rápido y radical de los procesos estratégicos de valor agregado y de los sistemas, las políticas y las estructuras organizacionales que los sustentan- para optimizar los flujos de trabajo y la productividad de una organización. Reingeniería de procesos es la actividad que analiza el funcionamiento y el valor de los procesos existentes en el negocio y 


\section{La administración de la salud y la calidad del desempeño para los \\ beneficiarios}

Vol. 2, núm. 4., (2018)

María A Cedeño Ugalde; Deysi Delgado López; Johanna Margarita García Quiroz; Sandra Jazmín Muñiz Tóala; Liliana Maribel Pionce Sánchez; Tatiana Lissette Marcillo Quimis

hace cambios radicales para mejorar espectacularmente sus resultados, a juicio del cliente. Klein y Manganelli, (2004)

La reingeniería se ocupa, fundamentalmente, del estudio de los procesos, teniendo en cuenta que es lógico suponer que en cualquier empresa o institución se realicen continuamente múltiples procesos o infinidad de estos. En la actualidad para el estudio de los sistemas se aplica el enfoque de procesos (proceso y resultado o producto) y se define de la forma siguiente: "Proceso es algo que está en constante movimiento y cambio, que utiliza diferentes insumos y posee una estructura que realiza un conjunto de actividades y acciones cuya finalidad es obtener un producto o servicio (resultados) de interés para el cliente", Ramos, (2011).

Reingeniería es la revisión fundamental y el rediseño radical de procesos para alcanzar mejoras espectaculares en medidas críticas y contemporáneas de rendimiento, tales como costos, calidad, servicio y rapidez. Hammer y Champy, (1994).

Es necesaria una transformación, una sociedad avanzada y democrática no puede prescindir de una Administración Pública ágil y capaz de gestionar lo público con eficiencia y transparencia. El mundo está cambiando y las Administraciones no siempre lo hacen al mismo ritmo, lo que puede provocar que estas se conviertan en un freno al desarrollo. Para evitarlo, es necesario cambiar enfoques, estructuras y prácticas propias de otros siglos que impiden responder a los ciudadanos tal y como estos demandan. PwC, (2012).

La reingeniería de los procesos se puede entender como una comprensión fundamental y profunda de los procesos de cara al valor añadido que tienen para los clientes, para conseguir un 


\section{La administración de la salud y la calidad del desempeño para los beneficiarios}

Vol. 2, núm. 4., (2018)

María A Cedeño Ugalde; Deysi Delgado López; Johanna Margarita García Quiroz; Sandra Jazmín Muñiz Tóala; Liliana Maribel Pionce Sánchez; Tatiana Lissette Marcillo Quimis

rediseño en profundidad de los procesos e implantar un cambio esencial de los mismos para alcanzar mejoras espectaculares en medidas críticas del rendimiento (costes, calidad, servicio, productividad, rapidez, ...) modificando al mismo tiempo el propósito del trabajo y los fundamentos del negocio, de manera que permita establecer si es preciso unas nuevas estrategias corporativas. Alarcón, (1998).

\section{Administración de los recursos humanos en los servicios sanitarios}

El objetivo de la administración de recursos humanos son las personas y sus relaciones dentro de las organizaciones. Sirve para mantener la organización productiva, eficiente y eficaz, a partir del uso adecuado de su recurso humano. Ya es un paso adelante percibir que las personas que trabajan en la organización representan su recurso crítico, o sea, aquel sin el cual ella no consigue realizar su trabajo. Esto es particularmente correcto en las organizaciones de salud que, además de depender de la tecnología, no consiguen aplicar esta tecnología sin contar con el recurso humano adecuado. Hoy se considera que el concepto más adecuado está en discutir administración de talentos, ofreciendo a las personas la oportunidad de desarrollarse mientras trabajan en la organización, Morales, (2013).

A la hora de desarrollar los procesos de gestión organizacional en las instituciones de salud hay que tener presente que existen herramientas fundamentales donde se integran todos los recursos de la organización: estructura, funciones, producción de servicios, tecnología y capital humano que con el uso adecuado de una comunicación efectiva y eficiente influyen de forma positiva en el logro de los objetivos de trabajo. Martín, y Segredo, (2012). 


\section{La administración de la salud y la calidad del desempeño para los \\ beneficiarios}

Vol. 2, núm. 4., (2018)

María A Cedeño Ugalde; Deysi Delgado López; Johanna Margarita García Quiroz; Sandra Jazmín Muñiz Tóala; Liliana Maribel Pionce Sánchez; Tatiana Lissette Marcillo Quimis

Para dar una idea genérica y tradicional respecto a cómo comprender la administración de recursos humanos, se puede decir que ella puede ser entendida como un subsistema de cualquier organización. Al final, son las personas quienes rechazan el trabajo en las organizaciones. Inclusive en aquellos casos en que existe una marcada substitución del elemento humano por máquinas, aun así hay personas que van a recoger lo que fue colocado en esas máquinas, verificar lo que fue hecho por ellas, prenderlas, apagarlas, y decidir cuándo deben ser accionadas, Morales, (2013).

De cierta forma, es por medio de la utilización del modelo de teoría general de sistemas que el término "recurso humano" se vuelve aceptable. Administración de recursos humanos es el proceso administrativo aplicado al acrecentamiento y conservación del esfuerzo, las experiencias, la salud, los conocimientos las habilidades etc., de los miembros de la organización en beneficio del individuo, de la propia organización y del país en general, Morales, (2013).

El comportamiento de un trabajador no es el resultado directo de los factores organizativos existentes sino que depende en buena medida de la valoración que el trabajador hace de estos factores en conjunto con las actividades, interacciones y otras experiencias del colectivo con la Institución, lo que es reflejado en los estudios realizados en el campo de la administración en relación con el clima, la cultura y el desarrollo organizacional., Segredo, (2011) y Gonçalves, (1997).

La Gestión Transparente y la Prestación de servicios, implica desarrollar procesos gerenciales eficientes y garantizar la calidad en la atención, por lo que las unidades administrativas del MSPAS deben desarrollar las competencias de su Recurso Humano para 


\section{La administración de la salud y la calidad del desempeño para los beneficiarios}

Vol. 2, núm. 4., (2018)

María A Cedeño Ugalde; Deysi Delgado López; Johanna Margarita García Quiroz; Sandra Jazmín Muñiz Tóala; Liliana Maribel Pionce Sánchez; Tatiana Lissette Marcillo Quimis

responder al reto que plantea la desconcentración y la descentralización y ser capaces de abordar oportuna, eficaz y eficientemente los problemas públicos de la salud, con criterios técnicos, administrativos y financieros, MSPAS, (1997).

Enfoque sistémico de la gestión orientada a Resultados, de los servicios públicos de salud es una transformación intencional hacia niveles superiores de desarrollo. Es un proceso de aprendizaje colectivo por el cual un sistema incrementa su habilidad y deseo de servir a sus miembros y a su contexto. El sistema es la disposición de organizaciones, personas, materiales y procedimientos asociados con una determinada función o resultado. Normalmente, un sistema se compone de insumos, procesos y productos/resultados. La gestión por resultados, busca lograr un aumento al valor que aportan a la sociedad, orientando todos los esfuerzos institucionales al logro de Resultados, basándose en la mejora de la producción de servicios que debe entregarse a la sociedad, MSPAS, (1997).

En salud pública la necesidad de alcanzar nuevos y mayores logros en la atención a la salud de las personas y de incrementar la satisfacción de la población y de los propios trabajadores de las instituciones de salud con el servicio que se brinda, obliga a contar con métodos transformadores que permitan identificar todo lo que influye, de forma positiva o negativa, sobre el rendimiento de las personas en el trabajo, Segredo, et al., (2012).

En los procesos está la razón del éxito o el fracaso de nuestra práctica, de la inversión, de los valores que tenemos tan altos hoy en día y tan marcados en el error. Para esto es necesario analizar y estandarizar lo que hacemos, tanto en lo asistencial como en lo administrativo, y tener las herramientas de solución de problemas que nos permitan enfrentar los conflictos con visión 


\section{La administración de la salud y la calidad del desempeño para los \\ beneficiarios}

Vol. 2, núm. 4., (2018)

María A Cedeño Ugalde; Deysi Delgado López; Johanna Margarita García Quiroz; Sandra Jazmín Muñiz Tóala; Liliana Maribel Pionce Sánchez; Tatiana Lissette Marcillo Quimis

de gerente y de administrador, la forma de realizar administración y gestión de gerencia de nosotros mismos, con el conocimiento que tenemos sobre nuestros procesos, Morales, (2013).

El cuidado de la salud es un negocio y, como todo negocio, necesita una buena administración para funcionar sin inconvenientes. Los administradores de servicios médicos y de salud, también llamados ejecutivos de la salud o administradores de atención de la salud, planifican, dirigen, coordinan y supervisan los servicios de cuidado de la salud. Estos trabajadores son especialistas a cargo de un departamento clínico específico o generalistas que administran la totalidad de las instalaciones o un sistema, Morales, (2013).

El desarrollo organizacional como proceso es considerado un flujo identificable de acontecimientos correlacionados que se mueven a lo largo del tiempo hacia una meta general. Se plantea que el desarrollo organizacional no es un punto, sino el camino a seguir donde se despliegan y evolucionan de forma interactiva las acciones que se emprenden en la institución en busca de una gestión eficiente, donde la cultura de la organización influye poderosamente en la conducta individual y de grupo. Torres, (2003).

La estructura y el financiamiento del cuidado de la salud están cambiando rápidamente. Los futuros administradores de servicios médicos y de salud deben estar preparados para ocuparse de la integración de los sistemas del cuidado de la salud, las innovaciones tecnológicas, un entorno cada vez más regulado, la reestructuración del trabajo y un foco cada vez mayor en el cuidado preventivo. Serán requeridos para mejorar la eficiencia en las instalaciones de atención sanitaria y la calidad del servicio que proporcionan, Morales, (2013). 


\section{La administración de la salud y la calidad del desempeño para los beneficiarios}

Vol. 2, núm. 4., (2018)

María A Cedeño Ugalde; Deysi Delgado López; Johanna Margarita García Quiroz; Sandra Jazmín Muñiz Tóala; Liliana Maribel Pionce Sánchez; Tatiana Lissette Marcillo Quimis

Es necesario, además, que se desarrolle una cierta institucionalización profesional, de modo que se cuente con alguna referencia externa, más o menos independiente, que sea capaz de delimitar los ámbitos de competencia profesional que se deben requerir a los salubristas y, desde luego, que se produzca una acreditación objetiva de la existencia de estas competencias, una acreditación que debe ser objeto de actualización periódica. No se trata, sin embargo, de cumplir un trámite porque el propósito es obtener legitimación social, Segura, (2002).

La legitimación social se adquiere mediante el reconocimiento expreso de que son unos profesionales los que pueden dar mejores respuestas a algunos problemas determinados, que se distinguen de otros profesionales por su competencia específica. De manera que el salubrista deje de ser percibido como un instrumento de la administración o un mero agente del poder. También habrá que estar atentos a los cambios sociales y a la progresiva mundialización en la que las redes se están convirtiendo en los nuevos instrumentos operativos de la política. Segura, (2002).

Los dirigentes sabrán traducir las ideas abstractas al campo de lo concreto, o sea, descubrir cuáles son las personas aptas/capacitadas/habilitadas para hacer aquello que la organización precisa. Por ejemplo, decir que se necesita enfermeros es muy vago, pues hay profesionales de esta categoría que desarrollaron competencias en salud pública y hay otros que se especializaron en áreas médico-quirúrgicas; están los que desempeñan mejor actividades asistenciales y otros cuya predisposición se inclina a la actuación administrativa. Morales, (2013).

Se le da gran importancia a la valoración del clima organizacional en las instituciones porque constituye un elemento esencial en el desarrollo de su estrategia organizacional 


\section{La administración de la salud y la calidad del desempeño para los \\ beneficiarios}

Vol. 2, núm. 4., (2018)

María A Cedeño Ugalde; Deysi Delgado López; Johanna Margarita García Quiroz; Sandra Jazmín Muñiz Tóala; Liliana Maribel Pionce Sánchez; Tatiana Lissette Marcillo Quimis

planificada, posibilitándole al directivo una visión futura de la organización y como elemento diagnóstico de la realidad cambiante del entorno, lo que permite identificar las necesidades reales de la misma en relación con el futuro deseado, para de esta forma trazar las acciones que deben iniciarse en el presente que permitan alcanzar la visión del futuro diseñado para la institución. Segredo, (2011).

Siempre se trata de enfermeros, pero saber qué características se desea para realizar determinada tarea, aunque solo sea para ofrecer entrenamientos compatibles, puede tornar más eficiente y eficaz la organización como un todo, al menos a largo plazo. Para un mejor entendimiento de la problemática del personal de las instituciones de Salud detallaremos los factores que debe tomar en cuenta por el área de Personal de las Clínicas y Hospitales: Variedad de profesiones y niveles socioeconómicos de los trabajadores, Niveles de identificación con la Organización, Vocación de servicio a pacientes, Diferenciación de roles y funciones, Importancia de las funciones que desempeña el personal, Necesidades individuales y grupales, Niveles de motivación y comunicación en la organización, Capacidad de liderazgo en la organización. Morales, (2013).

Personal de atención directa al paciente:

Aquí agrupamos aquellos profesionales que brindan la atención directa a los pacientes, y que son los que en mayor proporción responsables de la satisfacción del mismo, tomando en cuenta que son los que dan la cara al paciente y por lo tanto tiene que dar un trato amable y esmerado pues es lo que en realidad lo que percibe el paciente. Este grupo está compuesto por: 


\section{La administración de la salud y la calidad del desempeño para los beneficiarios}

Vol. 2, núm. 4., (2018)

María A Cedeño Ugalde; Deysi Delgado López; Johanna Margarita García Quiroz; Sandra Jazmín Muñiz Tóala; Liliana Maribel Pionce Sánchez; Tatiana Lissette Marcillo Quimis

Médicos, Odontólogos, Tecnólogos, Técnicos de laboratorio, Nutricionistas, Recepcionistas. Morales, (2013).

Personal de la Organización:

Son aquellos cuyo rol es el planificar el desarrollo de la institución en su conjunto, también su trabajo está centrado en brindar el soporte al funcionamiento de la organización, debe también de mantener la operatividad de la organización en su conjunto, su responsabilidad está orientada con la organización y no directamente con los pacientes que en ella se atienden, son los encargados de transmitir al resto de la organización la visión global de la empresa. El personal que conforma este tipo de trabajadores están: Administradores, Economistas, Ingenieros de Sistemas, Comunicadores, Profesionales de Marketing, Secretarias, Contadores. Morales, (2013).

Se entiende por Clima Organizacional al ambiente donde se reflejan las facilidades o dificultades que encuentra la persona para aumentar o disminuir su desempeño, o para encontrar su punto de equilibrio. Es decir, la percepción que tienen las personas, de cuáles son las dificultades que existen en una organización y la influencia que sobre estos ejercen las estructuras organizativas, factores internos o externos del proceso de trabajo actuando como facilitadores o entorpecedores del logro de la calidad de los objetivos de la organización. Segredo (2009).

La administración y el control de calidad en los servicios públicos

Lo que esperan los ciudadanos de un servicio público es la fiabilidad del trabajo que se entrega, la competencia de los servidores públicos, la capacidad de respuesta y la puntualidad, la 


\section{La administración de la salud y la calidad del desempeño para los \\ beneficiarios}

Vol. 2, núm. 4., (2018)

María A Cedeño Ugalde; Deysi Delgado López; Johanna Margarita García Quiroz; Sandra Jazmín Muñiz Tóala; Liliana Maribel Pionce Sánchez; Tatiana Lissette Marcillo Quimis

accesibilidad fácil y rápida a los trámites, información veraz en un lenguaje comprensible, la credibilidad y seguridad que garantice compromiso y confidencialidad, factores determinantes de la calidad de un servicio. Las buenas prácticas en la administración pública ejercida con calidad reducen los tiempos de espera y mejora la relación coste/rendimiento de los servicios públicos, incrementando la productividad con responsabilidad. Servicio de Acreditación Ecuatoriano, (2018).

El control es un elemento del proceso administrativo que incluye todas las actividades que se emprenden para garantizar que las acciones reales coincidan con las acciones planificadas. Todos los directivos de una organización tienen la obligación de controlar, de tal manera, el control es un elemento clave en la gestión organizacional, Romero, M., (2010).

La calidad de un servicio no solamente responde a requisitos normativos sino que se traduce en la satisfacción de los usuarios. Cuando hablamos de calidad en los servicios públicos, hablamos de la evolución que ha tenido toda la administración pública hacia la mejora continua, con el objetivo de optimizar las condiciones en que se entregan los servicios a los ciudadanos. Servicio de Acreditación Ecuatoriano, (2018).

Al hablar de calidad estamos hablando de una mayor operatividad, efectividad y responsabilidad social. Para lograr alcanzar estas metas, la evaluación es el único camino posible, ya que no se puede mejorar lo que no se conoce, y porque además, ofrece la posibilidad de participar y mejorar, al conocer en profundidad la realidad de las Organizaciones que la integran y que son prestadoras de los servicios, proporcionando a los usuarios mayor información sobre la Unidad de Organización, Casermeiro et al, (s/f). 


\section{La administración de la salud y la calidad del desempeño para los beneficiarios}

Vol. 2, núm. 4., (2018)

María A Cedeño Ugalde; Deysi Delgado López; Johanna Margarita García Quiroz; Sandra Jazmín Muñiz Tóala; Liliana Maribel Pionce Sánchez; Tatiana Lissette Marcillo Quimis

A partir de los resultados obtenidos por las evaluaciones, se podrán establecer planes de mejora o de calidad que producirán cambios profundos en la forma de gestionar las Organizaciones, ya que cada Unidad de Organización pasará a utilizar la información que le brinda su evaluación como el motor de su gestión. La importancia de la evaluación radica en que mejora el funcionamiento de las Organizaciones, ayuda a la creación de nuevas ideas y soluciones y fomenta la competencia por los recursos materiales y humanos, Casermeiro et al, $(\mathrm{s} / \mathrm{f})$.

Cualquier cambio puede ser manejado de dos maneras: oponiéndose permanentemente o liderándolo. Generalmente nos oponemos al cambio, lo que está es algo ya establecido y no hay forma de mejorarlo. El cambio hay que enfrentarlo y liderarlo con competitividad, y la calidad en salud es el elemento diferenciador que nos puede hacer competitivos. La calidad es un imperativo ético en salud, donde deben prevalecer las siguientes frases:

"hacer lo que hay que hacer y hacerlo bien"

"hacer correctamente las cosas correctas".

Día a día debemos recordar estas frases, muchas veces hacemos lo que tenemos que hacer pero mal hecho o hacemos lo que no tenemos que hacer bien hecho, Morales, (2013).

Analizando la evolución de la definición se puede establecer de dónde proviene la necesidad de ofrecer una mayor calidad del producto o servicio que se proporciona al cliente y, en definitiva, a la sociedad, en cualquier ámbito de actuación y cómo poco a poco se ha ido involucrando toda la organización en la consecución de este fin. Actualmente la calidad es un 


\section{La administración de la salud y la calidad del desempeño para los \\ beneficiarios}

Vol. 2, núm. 4., (2018)

María A Cedeño Ugalde; Deysi Delgado López; Johanna Margarita García Quiroz; Sandra Jazmín Muñiz Tóala; Liliana Maribel Pionce Sánchez; Tatiana Lissette Marcillo Quimis

requisito clave del producto o servicio así como un factor estratégico clave del que dependen la mayor parte de las organizaciones, para mantener su posición en el mercado asegurando su supervivencia. Llorens \& Fuentes, (2005).

Otra definición de calidad es: Un proceso de mejoramiento continuo, en donde todas las áreas de la empresa participan activamente en el desarrollo de productos y servicios, que satisfagan las necesidades del cliente, logrando con ello mayor productividad. Pérez, (2006).

La implementación de sistemas de calidad requiere de una participación integral de toda la institución, tomando en cuenta el alcance, implicaciones, procesos y estrategias para el posicionamiento de sus servicios, ofreciendo el mismo nivel de atención de forma constante y continua a lo largo del tiempo. El reto actual de los procesos de mejora de la calidad en la administración pública, no se centra en los objetivos estratégicos, la gestión y control de los procesos internos, sino en mejorar la calidad desde la demanda de los ciudadanos. Lo importante es saber cómo los usuarios de los servicios públicos ven y califican los mismos, y cómo quisieran que sea la atención y la eficacia. Servicio de Acreditación Ecuatoriano, (2018).

\section{Conclusiones.}

En la actualidad la administración de la salud, ha desarrollado un continuo y acelerado cambio, proyectado en la organización, ya que con los nuevos escenarios, exigencias y demandas de los diferentes sectores sociales y de la organización misma, debe responder ante la sociedad interviniendo y transformando su entorno marcando su desarrollo. Constituyéndose la 


\section{La administración de la salud y la calidad del desempeño para los beneficiarios}

Vol. 2, núm. 4., (2018)

María A Cedeño Ugalde; Deysi Delgado López; Johanna Margarita García Quiroz; Sandra Jazmín Muñiz Tóala; Liliana Maribel Pionce Sánchez; Tatiana Lissette Marcillo Quimis

administración sanitaria en el componente clave en la gestión que ha generado cambios cuya finalidad es lograr una mayor eficiencia en la Institución de salud, condición estratégica frente a la globalización.

La administración posee los elementos necesarios que aplicados al área de la salud, estos van a contribuir en el desarrollo organizacional de la institución de salud a fin de desarrollar una visión en conjunto del sistema optimizando los recursos económicos, humanos y técnicos con la intención de mejora el desempeño de la institución de salud hacia un crecimiento de calidad eficaz y eficiente.

Las instituciones de la salud tiene un reto importante puesto que son las que tiene que adaptarse a los procesos de transformación actual de esta forma el desempeño de la salud pública para los beneficiarios se concentre en modalidades de acción para mejorar la calidad del desempeño en las relaciones humanas, direccionando la política y las estrategias sanitarias a fin de lograr el servicio administrativo competente con el propósito de garantizar la excelencia de los servicios de salud en los diferentes niveles del sistema organizacional, para lo cual también se deben considerar los costos económicos por la prestación de los servicios, el desarrollo profesional de los recursos humanos prestadores de los servicios de salud científicos, profesionales y líderes que decidirán de las políticas económicas y públicas nacionales e internacionales. La estructura debe generar una mejor calidad orientada a competir, es la necesidad que tiene con el entorno globalizado. A fin de ampliar el nivel de satisfacción de los propios profesionales y la población. 


\section{La administración de la salud y la calidad del desempeño para los \\ beneficiarios}

Vol. 2, núm. 4., (2018)

María A Cedeño Ugalde; Deysi Delgado López; Johanna Margarita García Quiroz; Sandra Jazmín Muñiz Tóala; Liliana Maribel Pionce Sánchez; Tatiana Lissette Marcillo Quimis

\section{Referencias Bibliográficas}

Alarcón G., Juan Á., (1998), Reingeniería de procesos empresariales, FC Editorial, p.15.

Casermeiro, M., Scheuber, Y., Varas, D. y Contreras, A., (s/f), La Calidad en los Servicios

Públicos, Secretaria General, Oficina de Calidad de los Servicios, Gobierno de la

Provincia de Salta.

http://www.salta.gov.ar/descargas/archivos/ocspdfs/ocs_la_calidad_en_los_servicios_pub licos.pdf

Díaz, C., (1988), La teoría de delegación de autoridad, Universidad de la Habana, CENSAI.

Gonçalves, A., (1997), Dimensiones del Clima Organizacional, Disponible en: http://www.educadormarista.com/proyectoaprender/clima-organizacional.htm

Hammer, M. y Champy, J., (1994), Reingeniería, Editorial Norma, p.34.

Klein, Mark M. y Manganelli, Raymond L., (2004), Cómo hacer reingeniería, Editorial Norma, p.10.

Llorens Montes, F. J., \& Fuentes Fuentes, M. d., (2005), Gestión de la calidad empresarial.

Martín, X y Segredo, A., (2012), El capital humano en el desarrollo de las organizaciones, Escuela Nacional de Salud Pública, La Habana.

Minsal P., D. y Pérez R., Y., (2007), Hacia una nueva cultura organizacional: la cultura del conocimiento. Acimed, 16(3). Disponible en: http://bvs.sld.cu/revistas/aci/vool

Morales, J., (2013), La Administración en los servicios de salud, Tesis de post grado gerencia en salud, Universidad Metropolitana de Educación.

Mora V., C., (2005), El Capital Humano en el comportamiento organizacional, En GestioPolis.com. Disponible

en: http://www.gestiopolis.com/canales5/rrhh/elhucompor.htm

MSPAS, (1997), Modelo de atención integral en salud, Ministerio de Salud Pública y Asistencia Social.

https://www.paho.org/gut/index.php?option=com_docman\&view=download\&category_s lug=publications \&alias=378-modelo-de-atencion-mpas\&Itemid=518

Pérez, G., (2006), Gestiópolis, Calidad Total, http://www.gestiopolis.com 


\section{La administración de la salud y la calidad del desempeño para los beneficiarios}

Vol. 2, núm. 4., (2018)

María A Cedeño Ugalde; Deysi Delgado López; Johanna Margarita García Quiroz; Sandra Jazmín Muñiz Tóala; Liliana Maribel Pionce Sánchez; Tatiana Lissette Marcillo Quimis

PwC, (2012), ¿En qué hay que transformar la Administración Pública española?, Cómo afrontar el reto del cambio del sector público, PricewaterhouseCoopers S.L. "PwC", www.pwc.com

Ramos D., B., (2011), Control de calidad de la atención de salud, 2da edición, Editorial Ciencias Medicas, La Habana, Cuba.

Romero, M., (2010), El control como fase del proceso administrativo. En: Carnota Lauzán O., Biblioteca virtual para formación postgraduada de directivos del sector salud: ENSAP, La Habana

Segura, A., (2002). Salud pública, política y administración: ¿rehenes o cómplices?, Gac Sanit, $16(3): 205-8$

Segredo P., A., (2009), Caracterización del Sistema de Dirección en la Atención Primaria de Salud, Rev Cubana Salud Pública, 35(4): 78-109. Disponible en: http://scielo.sld.cu/scielo.php?script=sci_arttext\&pid=S086434662009000400009\&lng=p $\mathrm{t}$

Segredo P., A., (2011), La gestión universitaria y el clima organizacional, Educ Med Super, 25(2): 164-177. Disponible en: http://scielo.sld.cu/scielo.php?script=sci_arttext\&pid=S0864$21412011000200013 \& \operatorname{lng}=\mathrm{es}$

Segredo P., A., Martín L., X., Gómez Z., O., Lozada C., M., (2012), Gestión y desarrollo organizacional en Salud Pública, Editorial Ciencias Médicas, $\mathrm{N}^{\mathrm{o}} 15$, Infor. http://www.revinfodir.sld.cu/index.php/infodir/article/view/353/397

Servicio de Acreditación Ecuatoriano, (2018), Calidad en los servicios públicos, http://www.acreditacion.gob.ec/calidad-en-los-servicios-publicos/

Torres, S., (2003), Desarrollo Organizacional Disponible en: http://www.gestiopolis.com/recursos/documentos/fulldocs/ger1/desorgsug.htm 